Article

\title{
Prognostic value of resistance proteins in plasma cells from multiple myeloma patients treated with bortezomib
}

\author{
Paweł Robak ${ }^{1}$, Janusz Szemraj ${ }^{2}$, Damian Mikulski ${ }^{3}$, Izabela Drozdz ${ }^{4}$, Karolina Juszczak ${ }^{2}$, Dariusz Jarych ${ }^{5,6}$, \\ Małgorzata Misiewicz ${ }^{7}$, Kacper Kościelny ${ }^{3}$, Wojciech Fendler ${ }^{3}$, Tadeusz Robak ${ }^{7,8,}$
}

w:; 1 Department of Experimental Hematology, Medical University of Lodz, 93-510 Lodz, Poland; pawel.robak@umed.lodz.pl (P.R.);

2 Department of Medical Biochemistry, Medical University of Lodz, 92-215 Lodz, Poland; janusz.szemraj@umed.lodz.pl (J.S.); karolina.juszczak@stud.umed.lodz.pl (K.J.)

3 Department of Biostatistics and Translational Medicine, Medical University of Lodz, 92-215 Lodz, Poland; damian.mikulski@stud.umed.lodz.pl ;(D.M.); kacper.koscielny@stud.umed.lodz.pl (K.K.); wojciech.fendler@umed.lodz.pl (W.F.)

4 Department of Clinical Genetics, Medical University of Lodz, 92-213 Lodz, Poland; izabela.drozdz@umed.lodz.pl (I.D.)

5 Laboratory of Personalized Medicine, Bionanopark, Lodz 93-465, Poland;

6 Laboratory of Virology, Institute of Medical Biology, Polish Academy of Sciences, 93-232 Lodz, Poland. , Bionanopark, 93-465 Lodz, Poland; djarych@cbm.pan.pl (D.J.)

7 Department of Hematology, Medical University of Lodz, 93-510 Lodz, Poland; robaktad@csk.umed.lodz.pl (T.R.); malgorzata.misiewicz@umed.lodz.pl (M.M.)

8 Copernicus Memorial Hospital, Lodz, 93-513 Lodz, Poland; Poland

* Correspondence: robaktad@csk.umed.lodz.pl (T.R.)

Orcid Tadeusz Robak http://orcid.org/0000-0002-3411-6357

Orcid Dariusz Jarych http://orcid.org/0000-0003-1303-7163

Orcid Damian Mikulski https://orcid.org/0000-0002-2806-2583

\begin{abstract}
While multiple myeloma (MM) treatment with proteasome inhibitors and other agents yields encouraging results, primary and secondary resistance remains an emerging problem. An important factor in such treatment resistance is the overexpression of several proteins. The present study comprehensively evaluates the expression of POMP, PSMB5, NRF2, XBP1, CMAF and MAFb proteins in plasma cells isolated from the bone marrow of $39 \mathrm{MM}$ patients treated with bortezomib-based regimens using enzyme-linked immunosorbent assay (ELISA). The proteins were selected on the basis of previous laboratory and clinical studies in bortezomib treated MM patients. It was found that the expression of the investigated proteins did not significantly differ between bortezomib-sensitive and bortezomib-refractory patients. However, the expression of some proteins correlated with overall survival (OS); this was significantly shorter in patients with higher POMP expression (HR 2.8, 95\% CI: 1.1-7.0, $p=0.0277$ ) and longer in those with higher MAFB expression (HR 0.32, 95\% CI: 0.13-0.80, $p=0.0147$ ). Our results indicate that high expression of POMP and MAFB in MM plasma cells may serve as predictors of OS in MM patients treated with bortezomib-based regimens. However, further studies are needed to determine the role of these factors in effective strategies for improving anti-myeloma therapy.
\end{abstract}

Keywords: bortezomib; cMAF; MAFb; multiple myeloma; POMP; prognosis; PFS; survival; OS 


\section{Introduction}

Multiple myeloma (MM, plasma cell myeloma) is a hematological malignancy characterized by accumulation of malignant plasma cells (PC) in the bone marrow (BM), often resulting in bone lesions, hypercalcemia, infections, anemia, and the production of monoclonal immunoglobulin [1]. The disease accounts for about $1.8 \%$ of all cancers and $18 \%$ of all hematologic malignancies, with an annual incidence of 4.5-6 cases per $100,000[2,3]$. The median age at diagnosis is 69 years and median survival is around four or five years (Stat Fact Sheets SEER. Myeloma. http://seer.cancer.gov/statfacts/html/ mulmy.html. Accessed on June 10th, 2021).

The proteasome inhibitors (PI), bortezomib, carfilzomib and ixazomib play a key role in the treatment of $\mathrm{MM}$, and several new drugs from this group are undergoing clinical trials $[4,5]$. These agents are reversible inhibitors of the $26 \mathrm{~S}$ proteasome, which plays a critical role in the pathogenesis and proliferation of MM cells. Proteasome inhibitors act via various mechanisms, including by exerting direct effects on myeloma cells, and inhibiting the activity of cytokines as well as several adhesion molecules and angiogenesis. They are also known to inhibit the action of nuclear factor kappa B (NF-kB), which plays a key role in the survival and proliferation of MM cells. Proteasome inhibition causes a number of downstream effects, including the inhibition of NF- $\mathrm{B}$ signaling [6]. The resulting endoplasmic reticulum stress leads to an unfolded protein response, the downregulation of growth factor receptors, suppression of adhesion molecule expression and inhibition of angiogenesis.

Bortezomib is a boronic acid dipeptide that reversibly inhibits the $\beta 5$ subunit of proteasome and thus its chymotrypsin-like activity $[7,8]$. Bortezomib is approved for first-line treatment and in relapsed/refractory patients [9]. However, eventually after several courses of treatment, most patients show resistance to bortezomib, and most demonstrate multiple drug resistance. In addition, approximately $20 \%$ of patients exhibit primary resistance that determines lack of response to treatment [10,11]. Reports indicate overexpression of several proteins in MM plasma cells, and that this may be an important factor in the pathomechanism of bortezomib resistance [11-14]. In addition, proteomics-based techniques have highlighted the proteomic patterns specific to response and treatment outcome in MM patients, and have created a possibility for the implementation of marker-based individualized therapies [15,16].

The present study performs a comprehensive evaluation of the expression of six previously-described proteins in PCs which may influence the prognosis in MM patients. Samples were taken from the $\mathrm{BM}$ of $39 \mathrm{MM}$ patients treated with bortezomib-based regimens and the expression of the POMP, PSMB5, NRF2, XBP1, cMAF and MAFB proteins were determined using enzyme-linked immunosorbent assay (ELISA).

\section{Materials and Methods}

\subsection{Patients.}

A total of 39 MM patients treated at the Department of Hematology, Copernicus Memorial Hospital, Lodz, Poland, were included in the study. Their demographic, clinical and laboratory details are shown in Table 1. The patients were classified as either bortezomib-sensitive or bortezomib-refractory according to their response to bortezomib-based therapy $[17,18]$. Response to treatment and relapse/progression were classified based on International Myeloma Working Group (IMWG) criteria [19,20]. The patients refractory to bortezomib-based treatment demonstrated progressive MM during bortezomib therapy or at two months from the end of treatment [19-21].

The study was conducted according to good clinical and laboratory practice rules and the principles of the Declaration of Helsinki. The study protocol was approved by the ethics committee of the Medical University Lodz (No RNN/103/16/KE). Written informed consent was obtained from all patients for anonymous usage of their diagnostic routine sample for the scientific project. 


\subsection{Collection of MM cells}

Bone marrow samples were collected from $39 \mathrm{MM}$ patients before treatment with a bortezomib-based regimen. Plasma cells were isolated from fresh BM aspirate samples using CD138 microbeads (Miltenyi). Purity was verified by flow cytometry on the basis of CD38 and CD138 expression, and was higher than $80 \%$ for all samples. Purified plasma cells from all studied patients were stored in DMEM medium with 10\%DMSO 10\% FBS at $-80^{\circ} \mathrm{C}$ for further use.

\subsection{Determination of human protein level in plasma cells}

The expression of six human proteins (POMP, PSMB5, NRF2, XBP1, CMAF and MAFB in MM PCs was analyzed in all samples. Quantitative detection of human protein concentration in PCs was performed by solid-phase sandwich ELISA assay as previously described [22,23]. POMP (Biobool, Hong Kong), PSMB5 (MyBioSource, USA), NRF2 (ThermoFischer Scientific, USA), XBP1 (MyBioSource, USA), cMAF (ThermoFischer Scientific, USA) and MAFb (MyBioSource, USA) reagents were applied. Lysates from PC pellets were prepared using Qiagen All Prep DNA/RNA/protein kit, according to the manufacturer's protocol. Up to $7 \times 106$ cells were used for the isolation. After the DNA and RNA isolation, protein pellet was suspended in PBS (1×) buffer. The total protein concentration was determined by BCA kit; this value should not exceed $0.3 \mathrm{mg}$ for each sample. All samples were added to the appropriate wells, as well as standards to create a standard curve, with immobilized antibodies specific for human POMP, PSMB5, NRF2, $\mathrm{XBP1}$, cMAF and MAFB proteins, and incubated. All standards and samples were pipetted in triplicate to the plate. After incubation and washing, biotin-conjugated anti-human POMP, PSMB5, NRF2, XBP1, cMAF and MAFB antibodies were added and incubated. After washing away any unbound substances, biotinylated antibody, the horseradish peroxidase-conjugated streptavidin was pipetted to the wells. Unbound immunoglobulins were washed off. An enzyme-labelled anti-human globulin was then bound to the antigen antibody complex. After washing, the bound conjugate was developed with the aid of a substrate solution (TMB) to render a blue soluble product, which turns yellow after adding the acid stopping solution. The optical density (OD) was measured at $450 \mathrm{~nm}$ with wavelength correction at $620 \mathrm{~nm}$ by using an Thermo LabSystems Multiskan Ascent 354 Plate Reader (LabX, Canada).

A standard curve was generated using curve-fitting software. The standard curve was plotted as the relative OD450 of each standard solution (Y) vs. the respective concentration of the standard solution $(X)$. The target concentration of the samples, i.e. the relative OD 450, calculated as (the OD 450 of each well) - (the O D 450 of blank well) was interpolated from the standard curve. The concentrations of samples were calculated by the appropriate dilution factor.

\subsection{Statistical analysis}

Continuous variables were presented as mean \pm standard deviation (SD). For normally distributed data, the relationships between two groups of continuous variables were determined using the two-sided independent Student's t-test. Survival analysis was conducted using a Kaplan-Meier estimate with univariate and multivariate Cox's proportional hazards models, as well as the log-rank test. Examined protein level was divided by ACTB protein level to normalize values. Cutoff Finder was used to determine the optimal cut point for protein level [24]. The optimal cutoff determined by Cutoff Finder is defined as the point with the most significant (log-rank test) split for OS. For clinics, where most of the decisions are binary, it seems most appropriate to use a procedure based on the stratification of a continuous biomarker variable into two groups. Normalized protein expression values for all samples and most important clinical variables were provided in Table S1.

\section{Results}


The demographic, clinical, and laboratory characteristics of the MM patients enrolled for the study are presented in Table 1 . The mean age of the study cohort was $66.8 \pm 8.9$ years (range: 39 - 81). Ten patients had received at least one prior therapy before bortezomib-based regimen initiation. Twenty-three $(59.0 \%)$ patients displayed $\operatorname{IgG}$ paraprotein, eight $(20.5 \%)$ demonstrated $\operatorname{IgA}$, and nine $(23.1 \%)$ had light chain disease (LCD). Cytogenetics data were available for 20 patients (51.2\%): amp (1q) was the most common abnormality (28.2\%), followed by IGH rearrangements $(17.9 \%)$, followed by $\mathrm{t}(4 ; 14)(10.3 \%), \operatorname{del}(13 q)(5.1 \%)$ and $\operatorname{del}(17 p)(5.1 \%)$.

Table 1. The characteristics of the MM patients included in the analysis. Data shown as frequencies $\mathrm{N}(\%)$ unless otherwise specified.

\begin{tabular}{|c|c|}
\hline Characteristics & Overall $(\mathrm{N}=39)$ \\
\hline \multirow[t]{2}{*}{ Sex } & M: 23 (59.0) \\
\hline & F: $16(41.0)$ \\
\hline Age mean \pm SD & $66.8 \pm 8.9$ \\
\hline (range) & $(39-81)$ \\
\hline \multirow[t]{4}{*}{ ISS at diagnosis } & I: $12(30.8)$ \\
\hline & II: 7 (17.9) \\
\hline & III: 17 (43.6) \\
\hline & Data missing: 3 (7.7) \\
\hline \multicolumn{2}{|l|}{ Paraprotein } \\
\hline $\operatorname{IgG}$ & $23(59.0)$ \\
\hline LCD & $8(20.5)$ \\
\hline $\operatorname{Ig} \mathrm{A}$ & $8(20.5)$ \\
\hline $\mathrm{HB}<10 \mathrm{~g} / \mathrm{dL}$ at diagnosis & $14(35.9)$ \\
\hline Creatinine $>2 \mathrm{mg} / \mathrm{dL}$ at diagnosis & $4(10.3)$ \\
\hline Calcium $>2.5 \mathrm{mmol} / \mathrm{L}$ at diagnosis & $11(28.2)$ \\
\hline Bone disease & $20(51.3)$ \\
\hline $\mathrm{CRP}>5 \mathrm{mg} / \mathrm{L}$ & $16(41)$ \\
\hline $\mathrm{LDH}>240 \mathrm{U} / \mathrm{L}$ & $10(25.6)$ \\
\hline \multicolumn{2}{|l|}{ Cytogenetics* } \\
\hline $\operatorname{amp}(1 q)$ & $11(28.2)$ \\
\hline $\mathrm{t}(4 ; 14)$ & $4(10.3)$ \\
\hline $\operatorname{del}(13 q)$ & $2(5.1)$ \\
\hline $\operatorname{del}(17 p)$ & $2(5.1)$ \\
\hline $\mathrm{t}(11 ; 14)$ & $1(2.6)$ \\
\hline $\operatorname{del}(1 p)$ & $1(2.6)$ \\
\hline $\mathrm{t}(14 ; 16)$ & $0(0)$ \\
\hline$t(14 ; 20)$ & $0(0)$ \\
\hline IGH rearrangements & $7(17.9)$ \\
\hline Prior treatment & $10(25.6)$ \\
\hline \multicolumn{2}{|l|}{ Bortezomib regimen: } \\
\hline VCD & $30(76.9)$ \\
\hline VMP & $4(10.3)$ \\
\hline
\end{tabular}




\begin{tabular}{cc}
\hline VD & $4(10.3)$ \\
VTD & $1(2.6)$ \\
\hline ASCT & $18(46.2)$ \\
\hline RTx & $10(25.6)$ \\
\hline Response to treatment & $10(25.6)$ \\
CR & $10(25.6)$ \\
VGPR & $8(20.5)$ \\
PR & $5(12.8)$ \\
SD & $6(15.4)$ \\
PD & $12(30.8)$ \\
\hline Refractoriness to bortezomib
\end{tabular}

*Cytogenetics data were available for 20 patients (51.2\%).

Abbreviations: LCD- light chain disease; VCD- bortezomib, cyclophosphamide and dexamethasone; VMP- bortezomib, melphalan and prednisone; VTD- bortezomib, thalidomide, dexamethasone; VD- bortezomib and dexamethasone; ASCTautologous steam cell transplantation, RTx- radiotherapy, ISS- International Staging System.

Most of the patients $(76.9 \%)$ had received a VCD (bortezomib, cyclophosphamide, and dexamethasone) regimen, four (10.3\%) VMP (bortezomib, melphalan, and prednisone), one (2.6\%) VTD (bortezomib, thalidomide, and dexamethasone) and four received VD (bortezomib and dexamethasone). Eighteen patients (46.2\%) underwent ASCT. Data on response according to IMWG criteria to bortezomib-based therapy were available for $39(100 \%)$ patients. Ten patients achieved CR $(25.6 \%)$, ten $(25.6 \%)$ a very good partial response (VGPR) and eight (20.5\%) a partial response (PR). Overall, 27 of the 39 patients were bortezomib sensitive, 12 were refractory to bortezomib-based regimens.

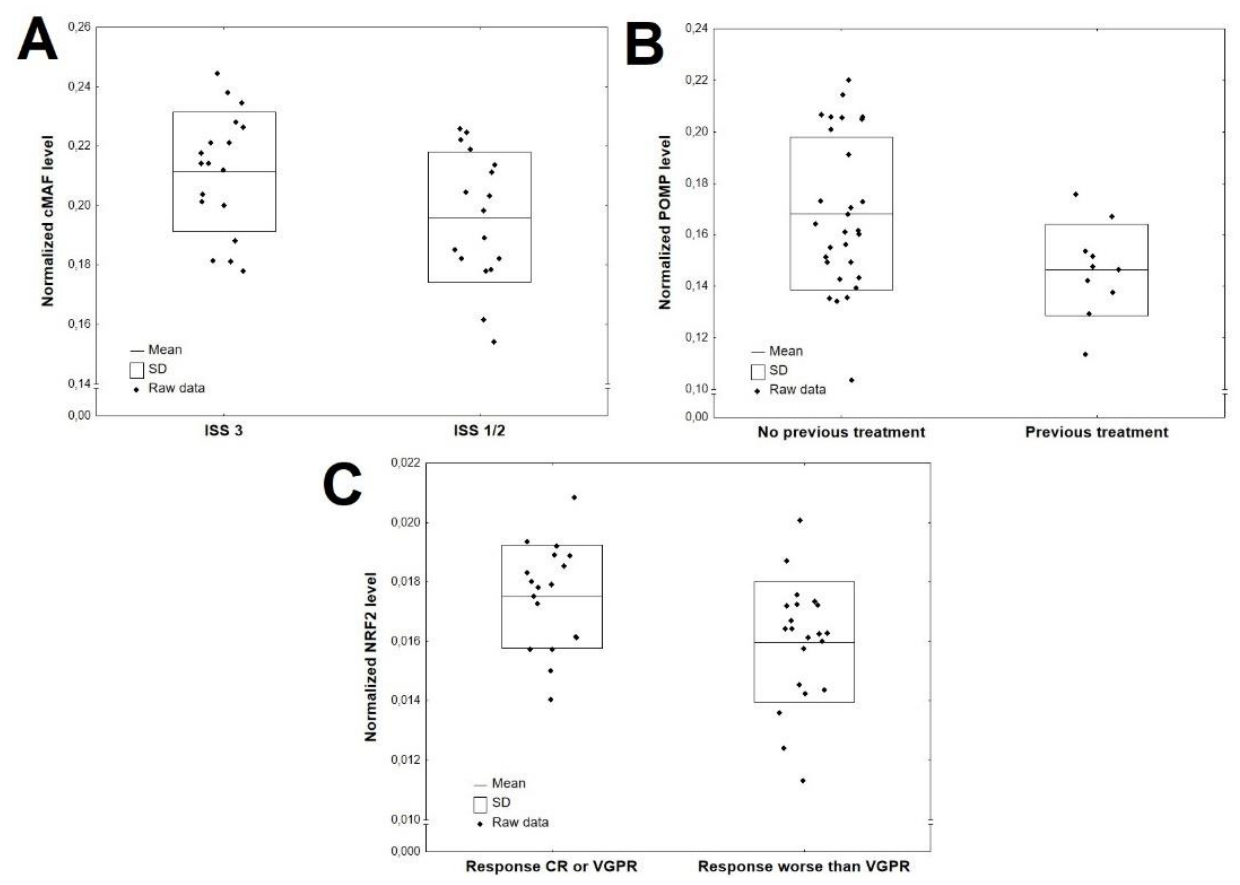

Figure 1. Significant differences in protein levels according to: (A) ISS: cMAF, $p=0.0383$; (B) previous treatment: POMP, $p=0.0350(\mathrm{C})$ response after bortezomib-based treatment: NRF2, $p=$ 0.0167 .

Comparisons of protein level according to International Staging System (ISS), previous treatment and CRAB symptoms- anaemia, hypercalcaemia, renal failure, and oste- 
olytic bone lesions are provided in Tables S2-S7 (Supplementary Materials). Generally, higher levels of $\mathrm{CMAF}(p=0.0383)$ were observed in patients with ISS stage III compared to stage I and II (Figure 1A, Table S2). POMP level was higher in patients without previous treatment ( $p=0.0350$, Figure 1B, Table S3). No significant difference was found between selected protein levels with regard to CRAB symptoms: anemia at diagnosis, hypercalcemia, renal failure, bone disease and bortezomib refractoriness (Tables S4-S7). NRF2 level was increased in patients with CR or VGPR response after bortezomib-based therapy compared to those with a worse response ( $p=0.0167$, Figure 1C, Table S8). No statistically significant differences in protein expression levels were observed between the bortezomib-refractory and bortezomib-sensitive groups (Table S9).

Data on progression-free survival (PFS) and overall survival (OS) was available for all patients. The median PFS was 11.6 (95\% CI: 8.1 - 13.3) months and the median OS was 28.2 (95\% CI: 21.0 - 34.2) months. Univariate Cox proportional hazards regression analysis was conducted to determine the prognostic value of the clinical variables and the normalized protein levels. The optimal cutpoints for normalized protein level dichotomization, determined by Cutoff Finder, are provided in Table S10.

ISS III (HR 2.12, 95\% CI: 1.07-4.19, $p=0.0308$ ) and previous treatment (HR 2.65, 95\% CI 1.18-5.91, $p=0.0178$ ) were associated with shorter PFS (Table 2). Autologous stem cell transplant (ASCT) in the treatment schedule (HR 0.35, 95\% CI: 0.17- 0.71, $p=0.0035$ ) and at least very good partial response $(\geq \mathrm{VGPR})$ to myeloma treatment $(\mathrm{HR} 0.47,95 \% \mathrm{CI}$ : $0.24-0.93, p=0.0292)$ were related with longer PFS. Protein levels did not impact PFS significantly. Statistically significant variables were entered to multivariate analysis using Cox's proportional hazards regression model with a stepwise selection procedure. The final model consisted of two variables: $\geq$ VGPR and ASCT (Table 3).

Table 2. Univariate Cox regression analyses for progression-free survival and overall survival.

\begin{tabular}{|c|c|c|c|c|c|c|c|c|c|c|}
\hline \multirow{3}{*}{ Variables } & \multicolumn{5}{|c|}{ PFS } & \multicolumn{5}{|c|}{ os } \\
\hline & \multirow{2}{*}{ Coefficient } & \multirow{2}{*}{$p$-value } & \multirow{2}{*}{$\mathrm{HR}$} & \multicolumn{2}{|c|}{$95 \% \mathrm{CI}$} & \multirow{2}{*}{ Coefficient } & \multirow{2}{*}{$p$-value } & \multirow{2}{*}{ HR } & \multicolumn{2}{|c|}{$95 \% \mathrm{CI}$} \\
\hline & & & & lower & higher & & & & lower & higher \\
\hline ISS III & 0.38 & 0.0308 & 2.12 & 1.07 & 4.19 & 0.34 & 0.1284 & 1.95 & 0.82 & 4.64 \\
\hline previous treatment & 0.49 & 0.0178 & 2.65 & 1.18 & 5.91 & 0.16 & 0.5265 & 1.36 & 0.52 & 3.56 \\
\hline$\geq$ VGPR & -0.38 & 0.0292 & 0.47 & 0.24 & 0.93 & -0.18 & 0.4102 & 0.69 & 0.29 & 1.66 \\
\hline ASCT & -0.53 & 0.0035 & 0.35 & 0.17 & 0.71 & -0.65 & 0.0074 & 0.27 & 0.10 & 0.70 \\
\hline Sex (M) & 0.12 & 0.4781 & 1.28 & 0.65 & 2.53 & 0.05 & 0.8298 & 1.10 & 0.45 & 2.69 \\
\hline $\mathrm{HB}<10 \mathrm{~g} / \mathrm{dL}$ & 0.12 & 0.4967 & 1.27 & 0.64 & 2.54 & 0.07 & 0.7431 & 1.16 & 0.48 & 2.81 \\
\hline Calcium > $2.5 \mathrm{mmol} / \mathrm{L}$ & -0.13 & 0.4838 & 0.77 & 0.38 & 1.59 & 0.01 & 0.9747 & 1.02 & 0.40 & 2.55 \\
\hline Creatinine $>2 \mathrm{mg} / \mathrm{dL}$ & -0.14 & 0.6125 & 0.76 & 0.27 & 2.17 & 0.22 & 0.482 & 1.57 & 0.45 & 5.46 \\
\hline Bone disease & 0.03 & 0.8753 & 1.06 & 0.54 & 2.06 & -0.06 & 0.7691 & 0.88 & 0.37 & 2.08 \\
\hline High POMP & -0.064 & 0.7434 & 0.880 & 0.410 & 1.889 & 0.515 & 0.0277 & 2.802 & 1.120 & 7.010 \\
\hline High PSMB5 & 0.059 & 0.7343 & 1.125 & 0.570 & 2.219 & 0.271 & 0.2319 & 1.720 & 0.707 & 4.188 \\
\hline High NRF2 & -0.205 & 0.2784 & 0.663 & 0.315 & 1.394 & -0.264 & 0.3104 & 0.590 & 0.213 & 1.635 \\
\hline High XBP1 & -0.047 & 0.7954 & 0.911 & 0.450 & 1.845 & -0.301 & 0.2419 & 0.548 & 0.200 & 1.501 \\
\hline High cMAF & 0.237 & 0.1972 & 1.608 & 0.781 & 3.310 & 0.280 & 0.0988 & 2.522 & 0.841 & 7.565 \\
\hline High MAFB & -0.270 & 0.1582 & 0.583 & 0.276 & 1.233 & -0.572 & 0.0147 & 0.319 & 0.127 & 0.798 \\
\hline
\end{tabular}

Table 3. Final multivariate Cox regression analyses for PFS and OS of MM patients.

Variables PFS




\begin{tabular}{cccccc}
\hline & \multirow{2}{*}{ Coefficient } & $p$-value & HR & \multicolumn{2}{c}{$95 \%$ CI } \\
\cline { 5 - 6 }$\geq$ VGPR & -0.43 & 0.0170 & 0.43 & 0.21 & 0.86 \\
\hline ASCT & -0.56 & 0.0021 & 0.32 & 0.16 & 0.66 \\
\hline \multicolumn{7}{c}{ OS } \\
\hline ASCT & -0.79 & 0.0025 & 0.20 & 0.07 & 0.57 \\
\hline High MAFB & -0.92 & 0.0005 & 0.16 & 0.06 & 0.45 \\
\hline High POMP & 0.60 & 0.0189 & 3.30 & 1.22 & 8.94 \\
\hline
\end{tabular}

The only clinical variable that significantly impacted OS was ASCT procedure (HR $0.27,95 \%$ CI: $0.10-0.70, p=0.0074)$. Among the proteins that significantly impacted OS in univariate analyses, high expression of POMP (HR 2.8, 95\% CI: 1.1-7.0, $p=0.0277$ ) was related with shorter OS, whereas high expression of MAFB (HR 0.32, 95\% CI: 0.13-0.80, $p$ $=0.0147)$ was associated with longer OS. The corresponding Kaplan-Meier plots are shown in Figure 2. Significant variables were entered to multivariate analysis using Cox's proportional hazards regression model with a stepwise selection procedure. The final multivariate model included all three significant variables: ASCT, high POMP expression and high MAFB expression (Table 3).

Figure 2. Kaplan-Meier plots for each of the significant proteins in the univariate analyses for OS: (A) POMP, (B) MAFB.
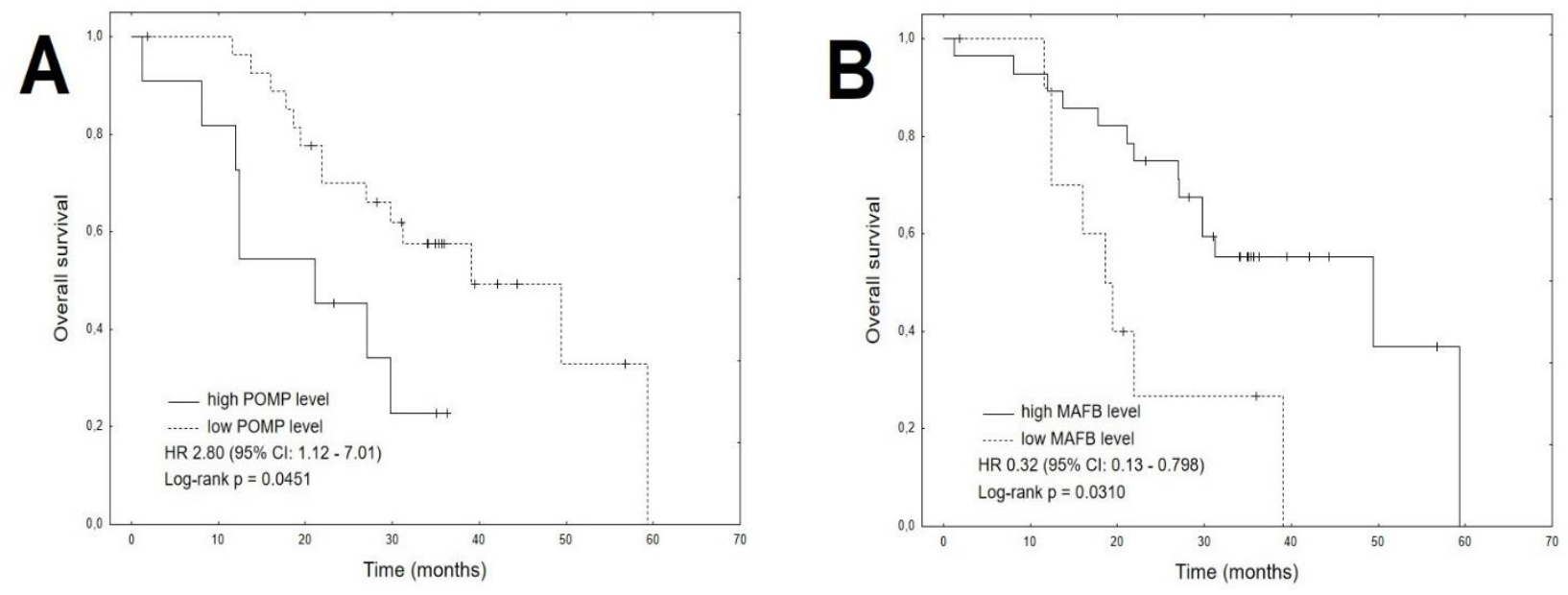

\section{Discussion}

The present study examined the levels of six proteins (POMP, PSMB5, NRF2, XBP1, cMAF and MAFB) in the PC from BM of 39 MM patients, treated with bortezomib-based regimens. ELISA was used to detect cellular protein levels. The proteins were selected on the basis of previous laboratory and clinical studies investigating their expression in bortezomib-sensitive and refractory MM patients and their influence on treatment results of bortezomib-based regimens.

None of the investigated proteins significantly differed between bortezomib-sensitive and bortezomib-refractory patients. However, the expression of some proteins correlated with OS. In particular, OS was significantly shorter in patients with higher expression of POMP $(p=0.0277)$ and longer in patients with higher expression of MAFB $(p=0.0147)$.

The MAFB and cMAF proteins are two key members in the MAF family. They share a similar structure, acting as transcription factors regulating gene transcription by cyclic adenosine monophosphate-response elements, specifically those including a DNA-binding domain and transcription activation domain [25]. cMAF is a critical oncogenic transcription factor influencing myelomagenesis [26]. Recent studies suggest that 
cMAF and MAFB proteins are substrates of USP5 (Ubiquitin specific peptidase 5) [27]. High MAFB protein expression is one of the most frequent oncogenic events in the progression of MM, and confers innate resistance to bortezomib [28]. Quiang et al. demonstrated that high MAFB protein expression is associated with resistance to proteasome inhibitors, bortezomib and carfilzomib in MM cell lines and that MAFB mediated proteasome inhibitor resistance [28]. However, in the present study, similar levels of MAFB were observed in BM plasma cells of bortezomib sensitive and bortezomib refractory patients.

Low expression of POMP in MM cells was also associated with longer OS. The POMP expression is essential for de novo biogenesis of proteasome, and its increased expression is one of the mechanisms of acquired resistance to PI [29]. There are also reports demonstrating that some cell lines resistant to bortezomib have increased expression of POMP [12,29]. It has been previously documented that serum POMP mRNA was significantly up-regulated in MM patients refractory to bortezomib-based treatment [30]. However, in the present study, bortezomib sensitive and refractory patients demonstrated similar levels of POMP expression in MM at the protein level, probably due to the relatively low number of patients.

In a study of cell lines (V10R) RPMI 8226, OPM-2, ANBL-6 and KAS-6/1, Li et al. report an increase in POMP protein expression in MM cells resistant to bortezomib [29]; they also found the suppression of POMP protein via shRNAs to restore cell sensitivity, while its over-expression in cells not subject to prior treatment increased resistance. The researchers also identified a protein-binding site for a suppressive factor, Nuclear factor erythroid 2-related factor 2 (NRF2) in the promoter region of the POMP protein. Although its increased expression should increase sensitivity to bortezomib, increased NRF2 levels were observed in resistant cells, together with increased levels of POMP protein. The level of activation of the two proteins varied between cell lines, and it appeared to have a stronger effect on bortezomib sensitivity in the KAS-6/1 than the OPM-2 line [29].

It was not possible to document the prognostic value of other proteins including PSMB5, NRF2 and XBP1. The proteasome subunit $\beta 5$ (PSMB5) is a protein encoded by the PSMB5 gene that contributes to the complete assembly of 205 proteasome complex. PSMB5 point mutations and $\beta 5$ subunit overexpression were the most frequent changes observed in the bortezomib resistant cell lines [31-33]. Nuclear factor erythroid 2-related factor 2 (NRF2) is a key regulator of MM cell survival in patients treated with PI [34]. NRF2 is constitutively activated in $50 \%$ of samples from MM patients, as well as several MM cell lines [34]. In addition, genetic inhibition of constitutively-expressed NRF2 reduced the viability of MM cells. Importantly PI increased the expression of NRF2 both in primary MM cells and in MM cell lines. Finally, inhibition of NRF2 in combination with PI treatment significantly increases apoptosis in MM cells [34]. XBP1 is an important transcription factor necessary for B cell differentiation into PCs, being responsible for the final maturation of plasmablasts to plasmocytes and induction of immunoglobulin secretion [35]. In a recent study, Brojan et al. found that bortezomib-resistant cells and BTZ-refractory MM patients exhibited lower sXBP1 levels [36]. These observations suggest that determination of sXBP1 levels prior to bortezomib treatment in MM may be useful to predict bortezomib resistance; however, these results were not confirmed in our present study.

The prognostic value of proteomic profiling has previously been reported by other authors $[14,15]$. Rajpal et al. used ELISA to validate the candidate protein biomarkers using unfractionated serum from 51 newly-diagnosed MM patients subsequently treated with thalidomide-based regimens [37]. Recently, Bai et al. analyzed the feasibility of predicting response to thalidomide-based therapy in previously-untreated $\mathrm{MM}$ using a novel panel of predictive serum markers; they used serum proteomic profiling to construct a MM model using four selected peptides evaluated in different disease states [38]. Western blot and ELISA were employed to validate the variability. The results demonstrate that a proteomics-based approach using a combination of immunodepletion, 2D-difference gel electrophoresis analysis, mass spectrometry and ELISA is an effective strategy for identi- 
fying proteins useful for predicting response to thalidomide. It was found that ZAG, VDB, SAA, B2M and Hp were demonstrated significantly different serum concentrations between thalidomide-refractory and thalidomide-sensitive patients.

However, in contrast to our present study, those given above used serum for proteomic profiling. In contrast, Dytfeld et al. performed a proteomic analysis of the PCs of MM patients subsequently treated with a proteasome inhibitor [15]. Their findings identify proteomic signatures that can be used to differentiate patients who achieved at least VGPR from those with a lower response to bortezomib-based chemotherapy. However, the study did not address any of the proteins included in the present analysis.

\section{Conclusions}

Lower expression of POMP and higher expression of MAFB correlated with longer OS in MM patients treated with borteomib. Evaluation of these proteins in plasma cells can be potentially useful in MM patients treated with bortezomib-based regimens. However, the clinical and biological significance of these findings needs further investigation.

Supplementary Materials: The following are available online at www.mdpi.com/xxx/s1, Table S1. Normalized protein expression values for all samples and most important clinical variables; Table S2. Normalized protein levels according to ISS stage. Data are presented as mean and SD; Table S3. Normalized protein levels according to previous treatment. Data are presented as mean and SD; Table S4. Normalized protein levels according to anemia at the diagnosis. Data are presented as mean and SD; Table S5. Protein levels according to hypercalcemia at the diagnosis. Data are presented as mean and SD; Table S6. Normalized protein levels according to renal failure at the diagnosis. Data are presented as mean and SD; Table S7. Normalized protein levels according to bone disease at the diagnosis. Data are presented as mean and SD; Table S8. Normalized protein levels according to the response to bortezomib-based chemotherapy according to the IMWG criteria. Data are presented as mean and SD; Table S9. Normalized protein levels according to refractoriness to bortezomib. Data are presented as mean and SD; Table S10. The optimal cutoff points for protein levels determined by Cutoff Finder.

Author Contributions: P.R., I.D, J.S., D.J., D.M. and T.R. designed the study and developed the methodology. P.R., J.S, I.D., C.P., K.J., and M.M. performed the experiments. P.R, D.M., K.K. and W.F. analyzed the data and performed statistical analyses; P.R., J.S., I.D., D.M. and T.R. drafted the manuscript. All authors read and approved the final manuscript.

Funding: This work was supported by a grant from the Narodowe Centrum Nauki (NCN - National Science Centre) (2016/23/B/NZ5/02529).

Institutional Review Board Statement: This study was approved by the Ethics Committee of the Medical University of Lodz (RNN/103/16/KE).

Informed Consent Statement: Informed consent was obtained from the patients included in the study.

Data Availability Statement: Normalized protein expression values for all samples and most important clinical variables were provided in Table S1. More data presented in this study are available from the corresponding author for request.

Acknowledgments: We thank Edward Lowczowski from the Medical University of Lodz for editorial assistance and Andrzej Berut for administrative support.

Conflicts of Interest: The authors declare no conflict of interest. The funders had no role in the design of the study; in the collection, analyses, or interpretation of data; in the writing of the manuscript, or in the decision to publish the results.

\footnotetext{
Abbreviations:

ASCT - autologous stem cell transplantation

ACTB - Beta-actin protein

$\mathrm{BM}$ - bone marrow

ELISA- enzyme-linked immunosorbent assay

LCD - light chain disease
} 


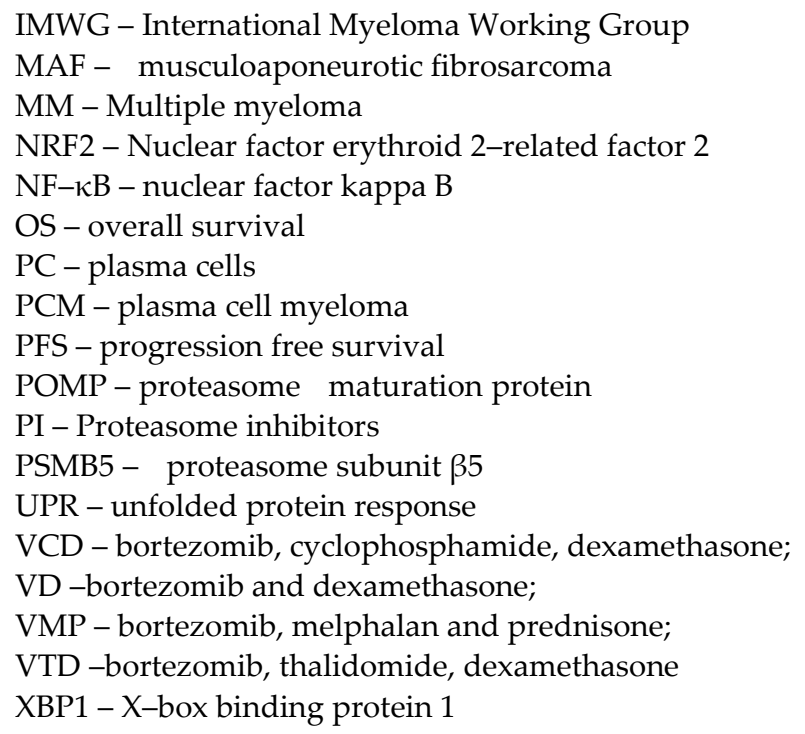

\section{References}

1. International Myeloma Working Group. Criteria for the classification of monoclonal gammopathies, multiple myeloma and related disorders: a report of the International Myeloma Working Group. Br. J. Haematol. 2003, 121,749-57.

2. Siegel, R.L.; Miller, K.D.; Jemal, A. Cancer statistics, 2020. CA Cancer J. Clin. 2020,70,7-30.

3. Gerecke, C.; Fuhrmann, S.; Strifler, S.; Schmidt-Hieber, M.; Einsele, H.; Knop, S. The diagnosis and treatment of multiple myeloma. Dtsch. Arztebl Int. 2016,113,470-476.

4. Robak, P.; Robak, T. Novel drugs for multiple myeloma. Topics in Anti-cancer Research. 2019, 8,1-43.

5. Gandolfi, S.; Laubach, J.P.; Hideshima, T.; Chauhan, D.; Anderson, K.C.; Richardson, P.G. The proteasome and proteasome inhibitors in multiple myeloma. Cancer Metastasis Rev. 2017,36,561-584.

6. Davis, L.N.; Sherbenou, D.W. Emerging therapeutic strategies to overcome drug resistance in multiple myeloma. Cancers (Basel). 2021,13,1686

7. Chen, D.; Frezza, M.; Schmitt, S.; Kanwar, J.; Dou, Q.P. Bortezomib as the first proteasome inhibitor anticancer drug: current status and future perspectives. Curr Cancer Drug Targets. 2011,11,239-253.

8. Robak, P.; Robak, T. Bortezomib for the treatment of hematologic malignancies: 15 years later. Drugs R D. 2019,19,73-92 .

9. Okazuka, K.; Ishida T. Proteasome inhibitors for multiple myeloma. Jpn. J. Clin. Oncol. 2018,48,785-793.

10. San Miguel, J.F.; Schlag, R.; Khuageva, N.K.; Dimopoulos, M.A.; Shpilberg, O.; Kropff , M.; Spicka, I.; Petrucci, M.T; Palumbo, A.; Samoilova, O.S.; et al. Bortezomib plus melphalan and prednisone for initial treatment of multiple myeloma. N. Engl. J. Med. 2008,359,906-917.

11. Robak, P; Drozdz, I.; Szemraj, J.; Robak, T. Drug resistance in multiple myeloma. Cancer Treat Rev. 2018,70,199-208.

12. Nikesitch, N.; Ling, S.C. Molecular mechanisms in multiple myeloma drug resistance. J. Clin. Pathol. 2016, 69,97-101

13. Gupta, N.; Sharma, A.; Sharma, A. Emerging biomarkers in multiple myeloma: A review. Clin. Chim. Acta. 2020, 503, 45-53.

14. Dytfeld, D.; Rosebeck, S.; Kandarpa, M.; Mayampurath, A.; Mellacheruvu, D.; Alonge, M.M.; Ngoka, L.; Jasielec, J.; Richardson, P.G.; Volchenboum, S.; et al. Proteomic profiling of naïve multiple myeloma patient plasma cells identifies pathways associated with favourable response to bortezomib-based treatment regimens. Br. J. Haematol. 2015, 170, 66-79.

15. Dytfeld. D.; Luczak, M.; Wrobel, T.; Usnarska-Zubkiewicz, L.; Brzezniakiewicz, K.; Jamroziak, K.; Giannopoulos, K.; Przybylowicz-Chalecka, A.; Ratajczak, B.; Czerwinska-Rybak, J.; et al. Comparative proteomic profiling of refractory/relapsed multiple myeloma reveals biomarkers involved in resistance to bortezomib-based therapy. Oncotarget. 2016, 7, 56726-56736.

16. Rajpal, R.; Dowling, P.; Meiller, J.; Clarke, C.; Murphy, W.G.; O'Connor, R.; Kell, M.; Mitsiades, C.; Richardson, P.; Anderson, K.C.; et al. A novel panel of protein biomarkers for predicting response to thalidomide-based therapy in newly diagnosed multiple myeloma patients. Proteomics. 2011, 11, 1391-402.

17. Robak, P.; Węgłowska, E.; Dróżdż, I.; Mikulski, D.; Jarych, D.; Ferlińska, M.; Wawrzyniak, E.; Misiewicz, M.; Smolewski, P.; Fendler, W.; et al. Cytokine and chemokine profile in patients with multiple myeloma treated with bortezomib. Mediators Inflamm. 2020,2020,1835836.

18. Robak, P.; Dróżdż, I.; Jarych, D.; Mikulski, D.; Węgłowska, E.; Siemieniuk-Ryś, M.; Misiewicz, M.; Stawiski, K.; Fendler, W.; Szemraj, J.; et al. The value of serum microRNA expression signature in predicting refractoriness to bortezomib-based therapy in multiple myeloma patients. Cancers (Basel). 2020, 12, E2569.

19. Durie, B.G.; Harousseau, J.L.; Miguel, J.S.; Bladé, J.; Barlogie, B.; Anderson, K.; Gertz, M.; Dimopoulos, M.; Westin, J.; Sonneveld, P. et al. International uniform response criteria for multiple myeloma. Leukemia 2006,20,1467-1473; 
20. Kyle, R.A., Rajkumar, S.V. Criteria for diagnosis, staging, risk stratification and response assessment of multiple myeloma. Leukemia 2009,23,3-9

21. Anderson, K.C.; Kyle, R.A.; Rajkumar, S.V.; Stewart A.K.; Weber, D.; Richardson, P.;ASH/FDA Panel on Clinical Endpoints in Multiple Myeloma. Leukemia. 2008, 22,231-239.

22. Pellett, S.; Tepp, W.H.; Johnson, E.A.; Sesardic, D. Assessment of ELISA as endpoint in neuronal cell-based assay for BoNT detection using hiPSC derived neurons. J. Pharmacol . Toxicol. Methods. 2017,88(Pt 1):1-6.

23. Yadirgi, G.; Stickings, P.; Rajagopal, S.; Liu, Y.; Sesardic, D. Immuno-detection of cleaved SNAP-25 from differentiated mouse embryonic stem cells provides a sensitive assay for determination of botulinum A toxin and antitoxin potency. J. Immunol. Methods. 2017, 451:90-99.

24. Budczies, J.; Klauschen, F.; Sinn, B.V.; Győrffy, B.; Schmitt, W.D.; Darb-Esfahani, S.; Denkert, C. Cutoff Finder: a comprehensive and straightforward Web application enabling rapid biomarker cutoff optimization. PLoS One. 2012, 7, e51862.

25. Eychene, A.; Rocques, N.; Pouponnot, C. A new MAFia in cancer. Nat. Rev. Cancer 2008, 8, 683-693.

26. Chen, X.H.; Xu, Y.J.; Wang, X.G.; Lin, P.; Cao, B.Y.; Zeng, Y.Y.; Wang, Q.; Zhang, Z.B.; Mao, X.L.; Zhang, T. Mebendazole elicits potent antimyeloma activity by inhibiting the USP5/c-Maf axis. Acta Pharmacol. Sin. 2019,40, 1568-1577.

27. Wang, S.; Juan, J.; Zhang, Z.; Du, Y.; Xu, Y.; Tong, J.; Cao, B.; Moran, M.F.; Zeng, Y.; Mao, X. Inhibition of the deubiquitinase USP5 leads to c-Maf protein degradation and myeloma cell apoptosis. Cell Death Dis. 2017, 8, e3058.

28. Qiang, Y.W.; Ye, S.; Huang, Y.; Chen, Y.; Van Rhee, F.; Epstein, J.; Walker, B.A.; Morgan, G.J.; Davies, F.E. MAFb protein confers intrinsic resistance to proteasome inhibitors in multiple myeloma. BMC Cancer. 2018, 18, 724

29. Li, B.; Fu, J.; Chen, P.; Ge, X.; Li, Y.; Kuiatse, I.; Wang H.; Wang, H.; Zhang, X.; Orlowski, R.Z. The Nuclear factor (Erythroid-derived 2)-like 2 and proteasome maturation protein axis mediate bortezomib resistance in multiple myeloma. J. Biol. Chem. 2015, 290, 29854-29868.

30. Robak, P.; Jarych, D.; Mikulski, D.; Dróżdż, I.; Węgłowska, E.; Kotkowska, A.; Misiewicz, M.; Smolewski, P.; Stawiski, K.; Fendler, W. et al. The prognostic value of whole-blood PSMB5, CXCR4, POMP, and RPL5 mRNA expression in patients with multiple myeloma treated with bortezomib. Cancers (Basel). 2021,13, 951.

31. Ri, M.; Iida, S.; Nakashima, T.; Miyazaki, H.; Mori, F.; Ito, A.; Inagaki, A.; Kusumoto, S.; Ishida, T.; Komatsu, H. et al. Bortezomib-resistant myeloma cell lines: a role for mutated PSMB5 in preventing the accumulation of unfolded proteins and fatal ER stress. Leukemia. 2010, 24, 1506-1512.

32. Oerlemans, R.; Franke, N.E.; Assaraf, Y.G.; Cloos, J.; van Zantwijk, I.; Berkers, C.R.; Scheffer, G.L.; Debipersad, K.; Vojtekova, K.; Lemos, C. et al. Molecular basis of bortezomib resistance: proteasome subunit beta5 (PSMB5) gene mutation and overexpression of PSMB5 protein. Blood 2008, 112, 2489-99.

33. Balsas, P.; Galán-Malo, P.; Marzo, I.; Naval, J . Bortezomib resistance in a myeloma cell line is associated to PSMB5 overexpression and polyploidy. Leuk. Res. 2012, 36, 212-218.

34. Sun, Y.; Abdul Aziz, A.; Bowles, K.; Rushworth, S. High NRF2 expression controls endoplasmic reticulum stress induced apoptosis in multiple myeloma. Cancer Lett. 2018, 412, 37-45.

35. Yang, Y.; Chen, Y.; Saha, M.N.; Chen, J.; Evans, K.; Qiu, L.; Reece, D.; Chen, G.A.; Chang, H. Targeting phospho-MARCKS overcomes drug resistance and induces antitumor activity in preclinical models of multiple myeloma. Leukemia. 2015, 29, 715-726

36. Borjan, B.; Kern, J.; Steiner, N.; Gunsilius, E.; Wolf, D.; Untergasser, G. Spliced XBP1 levels determine sensitivity of multiple myeloma cells to proteasome inhibitor bortezomib independent of the unfolded protein response mediator GRP78. Front. Oncol. 2020, 9, 1530.

37. Rajpal, R.; Dowling, P.; Meiller, J.; Clarke, C.; Mrphy, W.G.; O'Connor, R.; Kell, M.; Mitsiades, C.; Richardson, P.; Anderson, K.C. et al. A novel panel of protein biomarkers for predicting response to thalidomide-based therapy in newly diagnosed multiple myeloma patients. Proteomics. 2011;11, 1391-402.

38. Bai, J.; Yang, Y.; Wang, J.; Zhang, L.; Wang, F.; He, A. Variability of serum novel serum peptide biomarkers correlates with the disease states of multiple myeloma. Clin Proteomics. 2019 Apr 23;16:17. doi: 10.1186/s12014-019-9238-0. 